\title{
INTERFERENGE PHENOMENA IN DEFORMED SINGLE CRYSTALS OF ICE
}

\author{
By C. J. Readings* and J. T. Bartlett \\ (Meteorological Office, Bracknell, Berkshire, England)
}

\begin{abstract}
When rectangular single crystals of ice were subjected to uniaxial compression parallel to their long axes and viewed between crossed polarizers, interference fringes were often observed. Some of these interference bands were associated with grain boundaries formed as a result of "kinking". These can be explained in terms of the known anisotropic optical properties of ice and the change in the orientation of the optic axis across the boundary. This case has been analysed in detail with the aid of Jones' calculus and good quantitative agreement exists between the theory and the experimental observations.

Other interference bands were observed parallel to the trace of the basal plane on the surface of some deformed crystals. Alternative hypotheses for the explanation of this phenomenon have been considered and it seems probable that these bands are a result of slight random misorientations between adjacent slip lamellae. Applying Jones' calculus to a simple model of such a deformed crystal indicates that the required misorientations are of the order of $1^{\circ}$. If this explanation is correct, it implies that dislocations with non-basal Burgers vectors (probably $c[000 \mathrm{I}]$ ) make an active contribution to the deformation.

Resumé. Phénomìnes d'interférence dans uz monocristal déformé de glace. Lorsque des monocristaux rectangulaires de glace soumis à une compression uniaxiale parallèle à leurs grands axes sont observés entre des polariseurs croisés, des franges d'interférence sont souvent observées. Certaines de ces bandes d'interférence sont associées à des limites de grain qui se forment comme s'il y avait "nouage". On peut l'expliquer par les propriétés connues d'anisotropie optique de la glace et le changement dans l'orientation de l'axe optique à travers la limite. Ce cas a été analysé en détail avec l'aide du calcul de Jones et une bonne concordance quantitative existe entre la théorie et les observations expérimentales.

On observe d'autres bandes d'interférence parallèles à la trace des plans de base à la surface de quelques cristaux déformés. Une hypothèse ou une autre ont été considérées pour l'explication de ce phénomène et il semble probable que ces bandes sont le résultat de petites variations aléatoires d'orientation entre des plans de glissements voisins. L'application du calcul de Jones à un modèle simple d'un tel cristal déformé montre que les variations d'orientations nécessaires sont de l'ordre du degré. Si cette explication est correcte, elle implique que les dislocations avec des vecteurs de Burgers non basaux (probablement $c$ [ooo I]) prennent une part active à la déformation.
\end{abstract}

Zusammenfassung. Interferenzphänomene in deformierten Eis-Einkristallen. Wenn rechtwinklige, einkristalline Eisprismen parallel zu ihrer Längsachse komprimiert wurden, konnten oft Interferenzerscheinungen in polarisiertem Licht beobachtet werden. Einige dieser Interferenzbänder waren infolge der Stauchung mit der Bildung von Korngrenzen verbunden; sie können mit der bekannten optischen Anisotropie des Eises und dem Wechsel der optischen Achse an der Korngrenze erklärt werden. Dieser Fall wurde mit Hilfe der Matrizenrechnung nach Jones untersucht. Theorie und Experiment stimmen quantitativ gut überein.

Andere Interferenzbänder wurden parallel zur Schnittlinie der Basalebene mit der Oberfläche deformierter Eiskristalle beobachtet. Zwei verschiedene Hypothesen zur Erklärung dieses Phänomens wurden untersucht. Wahrscheinlich wurden die Interferenzbänder durch geringfügige zufällige Orientierungsunterschiede benachbarter Schichten in der Gleitzone verursacht. Die Matrizenrechnung nach Jones zeigt für ein einfaches Modell eines deformierten Kristalles, dass die erforderlichen Orientierungsunterschiede von Schicht zu Schicht etwa $1^{\circ}$ betragen müssen. Wenn diese Erklärung richtig ist, folgt daraus, dass Versetzungen mit Burgers-Vektoren ausserhalb der Basalebenen (wahrscheinlich $c$ [ooor]) aktiv an der Deformation beteiligt sind.

\section{Introduction}

Normal ice is a uniaxial crystal with hexagonal symmetry in which the optic axis is perpendicular to the basal plane (OOOI). This means tha changes which occur in the orientation of the $c$-axis of an ice crystal when it is deformed can usually be easily detected by placing it between crossed polarizers and noting variations in the extinction position. This property of ice has been used by a number of workers, notably Nakaya (1958) in his work on the bending of ice crystals and Higashi and Sakai (196I) in their experiments on the movement of small-angle grain boundaries under stress.

In the series of experiments described in this paper and briefly reported in a previous publication (Bartlett and Readings, 1968) large single crystals of ice were grown from pure water by a modified Bridgman technique. Rectangular specimens $(c .5 \mathrm{~cm} \times 2 \mathrm{~cm} \times 0.5 \mathrm{~cm})$

* Present address: Microbiological Research Establishment, Porton Down, Salisbury, Wiltshire, England. 
were cut from the single crystals and compressed parallel to their long axes in an apparatus which is described and illustrated in an earlier paper (Readings and Bartlett, ig68, especially fig. 3). During deformation the specimens were immersed in white paraffin (usually called "kerosene" in the United States) in order to prevent evaporation from their surfaces. The paraffin was contained in a vessel which had strain-free glass walls and was mounted between crossed sheets of "Polaroid", so that the specimens could be viewed in polarized light.

In addition to changes in the extinction positions which occurred when specimens were deformed, there were several situations in which interference bands were observed and these are the subject of this paper.

\section{INTERFERENCE BANDS AT GRAIN BOUNDARIES}

\section{I Observations}

Heavily deformed specimens often divide into two or more distinct ice crystals which are separated by well-defined grain boundaries, as described in our previous paper (Readings and Bartlett, I968). In many cases light and dark bands parallel to the trace of the grain

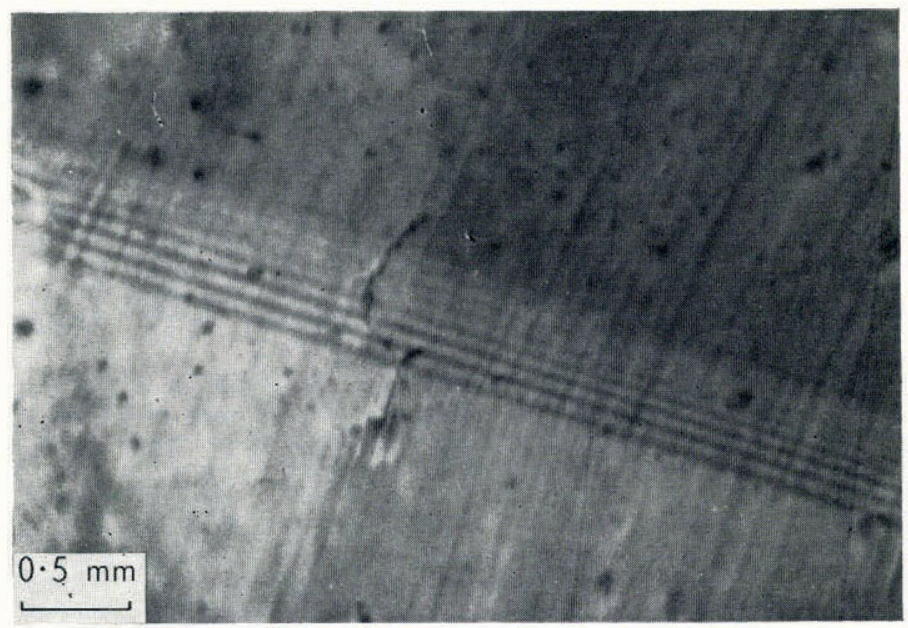

Fig. I. Interference bands at a grain boundary.

boundary on the surface of the specimen were then seen (Fig. I). These bands were only visible in polarized light and became coloured when the monochromatic sodium lamp used as a light source in most of our experiments was replaced by an unfiltered mercury lamp. It is therefore clear that they were an interference phenomenon.

It was also found that these bands appeared to lie between the traces of the grain boundary on the front and back surfaces of the specimen, but that their precise position and spacing generally altered as the polarizer and analyser were rotated together.

\subsection{Theory}

An explanation for this phenomenon can be given with reference to Figure 2. Plane polarized light entering a single crystal of ice will, in general, be divided into two components, polarized parallel to the "fast" and "slow" axes respectively. On emerging from the crystal these two components will have a phase difference $\delta$, and will recombine to give, in general, an elliptically polarized beam of light. The phase difference $\delta$ is called the retardance of the crystal and is given by $2 \pi\left(n^{\prime}-n_{0}\right) t / \lambda$, where $n^{\prime}$ and $n_{0}$ are the refractive indices for light 
polarized parallel to the fast and slow axes respectively, $t$ is the thickness of the crystal and $\lambda$ is the wavelength of the incident light in vacuo.

Now in the region of the grain boundary, $\mathrm{AB}$, the incident light will be divided into two components with a phase difference $\delta_{\mathrm{I}}$ by the first crystal and these will be further subdivided by the second crystal, which has a retardance $\delta_{2}$. Clearly $\delta_{1}$ and $\delta_{2}$ depend on the thicknesses of the two grains, $t_{1}$ and $t_{2}$ respectively, and therefore the state of polarization of the emergent beam will depend on its position relative to the grain boundary. For certain values of $\delta_{\mathrm{I}}$ and $\delta_{2}$, the emergent beam will be plane polarized parallel to the incident beam, and under these conditions a dark band will be observed when the specimen is viewed through the analyser.

Since the difference between the two refractive indices for ice is small $\left(n_{\mathrm{e}}=\mathrm{I} \cdot 3 \mathrm{IO} 4\right.$, $n_{0}=\mathrm{I} .3090$ for $\lambda=5893 \AA$ ) a ray of light passing through the grain boundary will be practically undeviated. Even for the extreme case in which the grain boundary is very steeply inclined to the surface and the difference between the appropriate refractive indices is nearly equal to the difference between the principal refractive indices, the deviation is less than about $2.5^{\circ}$. It is also reasonable to assume that the surfaces of the specimen remain very nearly normal to the incident beam and therefore the two parts of the crystal may be treated as two linear retarders in series.

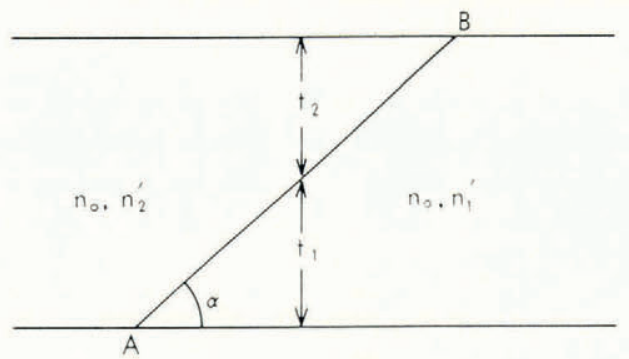

Fig. 2. Diagrammatic cross-section through a grain boundary.

Systems of non-absorbing retarders, rotators, and polarizers can be analysed conveniently by a matrix calculus introduced by Jones (r94 I [a], [b]; Hurwitz and Jones, I94I). In this calculus, the properties of a complex optical system are represented by a $2 \times 2$ matrix which is the product of a set of matrices, each of which corresponds to one component of the system. The electric vector $E_{\mathrm{t}}$ of light transmitted through any set of linear retarders, rotators, or polarizers can be represented by

$$
E_{\mathrm{t}}=M_{n} \cdot M_{n-1} \ldots \cdot M_{2} \cdot M_{1} E_{\mathrm{i}}
$$

where $M_{1}, M_{2}, \ldots, M_{n}$ are the $2 \times 2$ matrices representing the 1 st, 2 nd, . ., and $n$th components of the system encountered by the light and $E_{\mathrm{i}}$ is the electric vector of the incident light. If we are only concerned with relative intensities (as in the problem under discussion), certain simplifications can be made in the form of the appropriate matrices and vectors. In this case a linear retarder can be represented by

$$
R=\left[\begin{array}{rr}
\mathrm{e}^{\mathrm{i} \gamma} & 0 \\
0 & \mathrm{e}^{-\mathrm{i} \gamma}
\end{array}\right]
$$

where $2 \gamma$ is the retardance of the element and the $x$ - and $y$-axes are chosen to be parallel to its principal axes. 
If the axes of the retarder make any other angle $\theta$ with the $x$ - and $y$-axes, it can be represented by a matrix $R^{\prime}$, where

with

$$
R^{\prime}=S(\theta) R S(-\theta)
$$

$$
S(\theta)=\left[\begin{array}{cc}
\cos \theta & -\sin \theta \\
\sin \theta & \cos \theta
\end{array}\right] .
$$

Now consider the system of two retarders (retardances $2 \gamma_{1}$, and $2 \gamma_{2}$ ) situated between crossed polarizers which corresponds to a deformed ice crystal. Let the $x$ - and $y$-axes be defined by principal axes of the polarizer and analyser and let the principal axes of the retarders make angles $\theta_{1}$ and $\theta_{2}$ with the $x$ - and $y$-axes.

Then for this system

$$
E_{\mathrm{t}}=A\left\{S\left(\theta_{2}\right) R_{2} S\left(-\theta_{2}\right)\right\}\left\{S\left(\theta_{\mathrm{I}}\right) R_{\mathrm{I}} S\left(-\theta_{\mathrm{I}}\right)\right\} E_{\mathrm{i}}
$$

where $A$ is the matrix representing the analyser and is given by

$$
A=\left[\begin{array}{ll}
0 & 0 \\
0 & 1
\end{array}\right] \text {. }
$$

If the incident light is plane polarized, $E_{\mathrm{i}}=(\mathrm{I}, \mathrm{o})$, since only the relative intensities are important. Multiplication of the matrices then shows that the transmitted light vector has the form $E_{\mathrm{t}}=\left(\mathrm{o}, E_{y}\right)$ where

$$
E_{y}=\sin 2\left(\theta_{1}-\theta_{2}\right) \sin \gamma_{1} \sin \gamma_{2}+\mathrm{i}\left\{\sin 2 \theta_{1} \sin \gamma_{1} \cos \gamma_{2}+\sin 2 \theta_{2} \cos \gamma_{1} \sin \gamma_{2}\right\}
$$

and the observed intensity $I$ is equal to $E_{y \mathrm{r}^{2}}+E_{y \mathrm{i}^{2}}$ where $E_{y}=E_{y \mathrm{r}}+\mathrm{i} E_{y \mathrm{i}}$.

The general expression for the observed intensity can be simplified considerably if the polarizers are set so as to give complete extinction for one of the crystals. For example, if the first crystal appears dark, i.e. $\theta_{\mathrm{I}}=0$ or $\pi / 2$, the observed intensity in the region of the grain boundary becomes

$$
I=\sin ^{2} 2 \rho \sin ^{2} \gamma_{2}
$$

where $\rho$ is the angle between the principal axes of the two crystals.

The intensity is zero if $\rho=0$ or if $\gamma_{2}=m \pi$, where $m$ is an integer. The case $\rho=0$ is trivial, since it means that the extinction positions are the same for both crystals.

In the other case

$$
\gamma_{2}=\left(\pi t_{2} / \lambda\right)\left(n_{2}{ }^{\prime}-n_{0}\right)=m \pi
$$

where $n_{2}{ }^{\prime}$ and $n_{0}$ are the refractive indices appropriate to the second crystal. This means that there will be a dark band when $t_{2}=m \lambda /\left(n_{2}{ }^{\prime}-n_{0}\right)$ and if the angle between the grain boundary and the surface is $\alpha$, the observed spacing is given by

$$
s_{2}=\frac{\lambda \cot \alpha}{\left(n_{2}^{\prime}-n_{0}\right)} \text {. }
$$

Similarly the observed spacing will be $s_{1}$, when the second crystal is in the extinction position where

$$
s_{\mathrm{I}}=\frac{\lambda \cot \alpha}{\left(n_{\mathrm{I}}{ }^{\prime}-n_{0}\right)}
$$

\subsection{Experimental tests}

The formulae derived above were checked experimentally for the special case in which "kinking" was confined to the plane of the specimen. In such cases the inclination of the optic axis to the plane of the specimen did not change. Hence the spacing of the interference bands could be computed by using experimental values for the thickness of the specimen, 
INTERFERENCE PHENOMENA IN DEFORMED SINGLE CRYSTALS OF ICE 273

the inclinations of the optic axis to the surface, and the apparent width of the grain boundary. The spacings computed in this way gave very satisfactory agreement with the observed spacings as shown in Table I. Note that the angle between the slip traces in the grains and the angle between the extinction positions would be the same if kinking had actually occurred exactly in the plane of the specimen. Hence the last two columns in the table are a measure of the extent to which this criterion was satisfied.

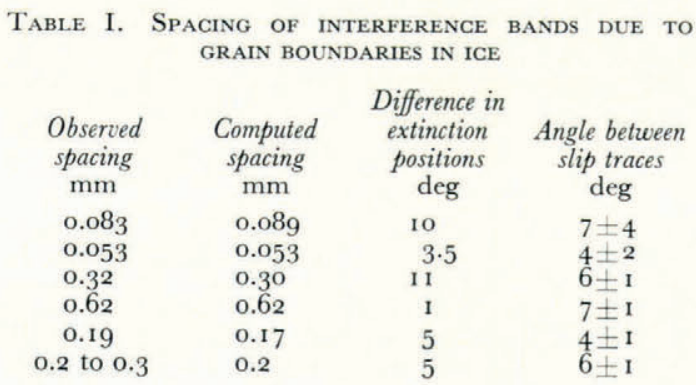

It is clear from the preceding discussion that it is not possible to resolve the interference bands if the inclination of the grain boundary to the surface is too great. In the particular case for which the optic axis is perpendicular to the direction of viewing, the spacing will be less than 0 . I mm (the approximate limit of resolution) if $\alpha \geqslant 75^{\circ}$. In any other case, the spacing will be below the limit of resolution for even smaller values of $\alpha$. This probably explains why the phenomenon was not observed at every grain boundary.

On some occasions irregular spacings were observed and this may be taken as andication that the grain boundaries themselves are sometimes curved or irregular.

\section{Interference bands Parallel to Slip Lines}

\section{I Observations}

In addition to the interference bands at grain boundaries which have already been described, light and dark bands parallel to the slip lines were frequently observed. An example of this type of band is shown in Figure 3.

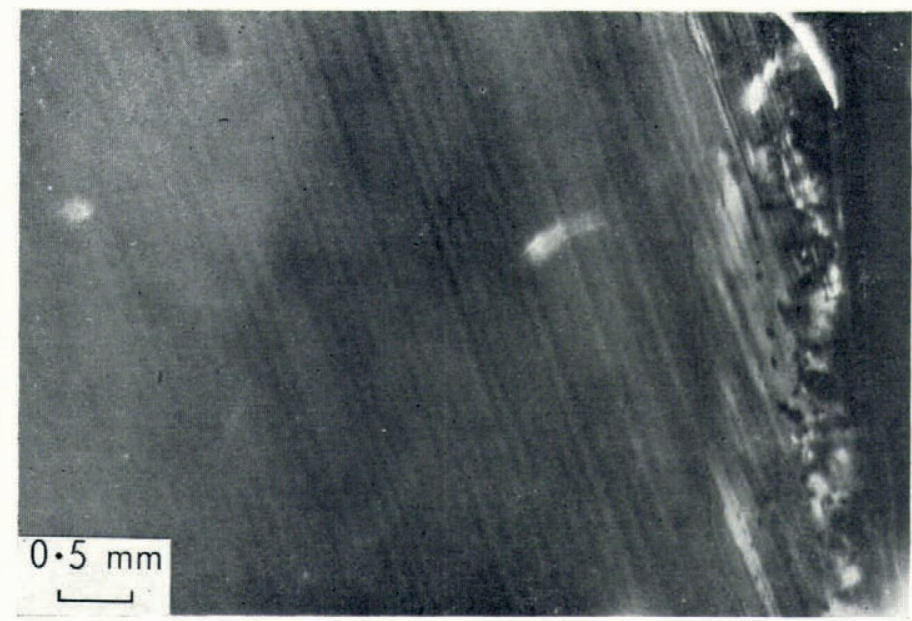

Fig. 3. Interference bands parallel to slip lines. 
These bands were first seen after a finite deformation which varied from specimen to specimen, but was generally less than the deformation required to produce a visible slip line. As the deformation proceeded they became more distinct and were usually found to be most prominent in those regions of the crystal in which the slip lines eventually became most marked. It is interesting to note, however, that even when most strongly developed, these interference bands could only be seen clearly near the extinction position for the undeformed crystal.

Although the interference bands were predominantly parallel to the trace of the basal plane in the surface, a few occasions were noted on which they changed direction. These could, however, generally be associated with changes in the thickness of the specimen and it was found that they could be bent by deliberately "contouring" a specimen, i.e. by melting part of it so that it had a variable thickness. This effect is shown in Figure 4.

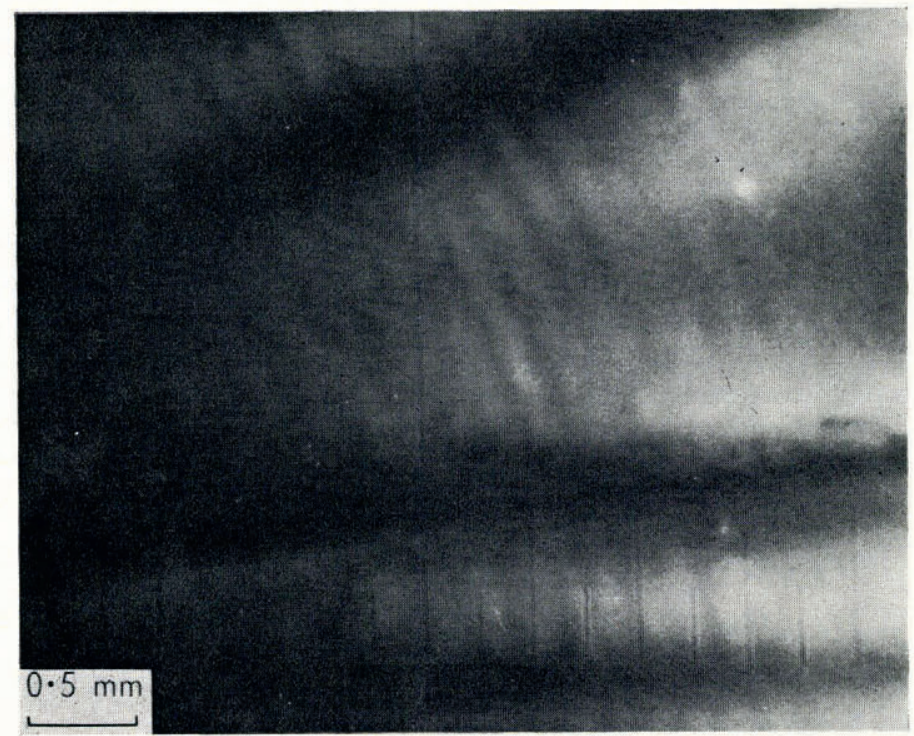

Fig. 4. Interference bands changing direction in a specimen of variable thickness. The broad dark bands radiating from the teft are fringes associated with the change in thickness.

The spacing of the bands was measured by taking photographs of some of the specimens. Although there were considerable variations in the observed spacings on any given specimen, a number of conclusions could be drawn. First, the spacing of the bands appeared to be independent of the wavelength of light used to view the specimen, the total strain and the orientation of the crossed polarizers. Secondly, changing the applied stress during deformation did not produce any observable change in the spacing, though the bands became more diffuse if the stress was removed. Thirdly, the spacing tended to increase as the inclination $\phi$ of the optic axis to a plane normal to the direction of viewing increased. This last result is demonstrated in Figure 5, in which the observed spacing is plotted as a function of $\phi$.

A direct comparison between the spacing of the interference bands and the spacing of the slip lines was not possible because they could not be measured accurately at the same time. However, earlier experiments (Readings and Bartlett, I968) showed that thicknesses of the slip lamellae are typically about $5^{\mathrm{O}-1} 5^{\mathrm{O}} \mu \mathrm{m}$ and therefore the slip-line spacings which correspond to lamellae of these thicknesses have also been plotted in Figure 5. It is clear from this diagram that the interference bands generally have a spacing about two or three times greater than the spacing of the corresponding slip lines. 
One other feature of the interference bands was that they were not normally visible when the inclination of the optic axis $\phi$ was less than about $15^{\circ}$ or greater than about $70^{\circ}$. This property is clearly demonstrated in Figure 6, in which the occurrence of interference bands is indicated as a function of $\phi$ and the angle $\psi$ between the optic axis and the axis of compression.

On a few occasions interference bands were visible in a deformed specimen when the angle $\phi$ was slightly greater than about $15^{\circ}$, but disappeared when the specimen kinked so that $\phi$ decreased. This observation would seem to indicate that the occurrence of interference bands depends on the direction of viewing and is not a consequence of different modes of deformation for specimens cut in different orientations.

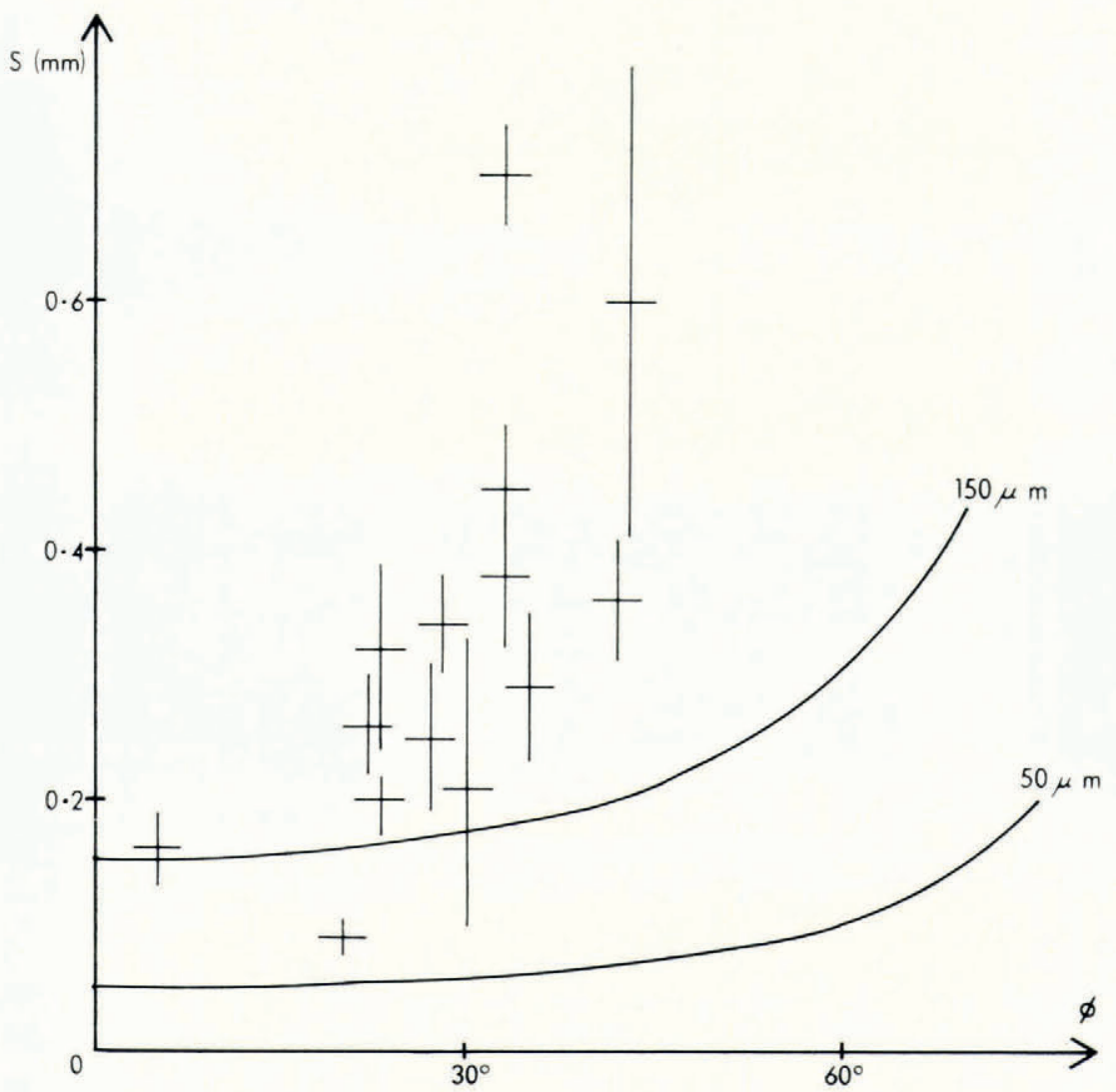

Fig. 5. Dependence of the observed spacing s of interference bands on the inclination of the optic axis $\phi$. The length of the vertical lines indicates the spread in the spacings observed on individual specimens.

\subsection{Theory}

From the observations described in the last paragraph, it is clear that a satisfactory theory of this phenomenon should account not only for the appearance of light and dark bands parallel to the slip lines which become more marked as deformation proceeds, but should also explain why they have the following properties.

(i) The spacing of the bands is independent of the orientation of the crossed polarizers and the wavelength of light used to view the crystal.

(ii) The spacing of the bands is usually a few times greater than the corresponding slip band spacing. 
(iii) The direction of the bands varies in specimens of variable thickness.

(iv) The bands are not normally visible when the optic axis is nearly parallel to, or nearly perpendicular to, the direction of viewing.

We shall consider two possible explanations for the phenomenon in some detail. The first suggestion is that the bands are produced as a result of changes in the optical properties of ice under stress. In this case the explanation would be similar to that given by Nye ( 949 , 1950) for the light and dark bands which he observed in deformed silver chloride crystals. We shall refer to this idea as the stress-optical hypothesis.

The second hypothesis is suggested by analogy with the interference bands observed at grain boundaries and discussed above (section 2). These bands are a consequence of the different orientations of the optic axis in two grains and it is therefore suggested that the interference bands under consideration arise from slight differences in the orientation of the optic axis in adjacent slip lamellae. We shall refer to this idea as the misorientation hypothesis.

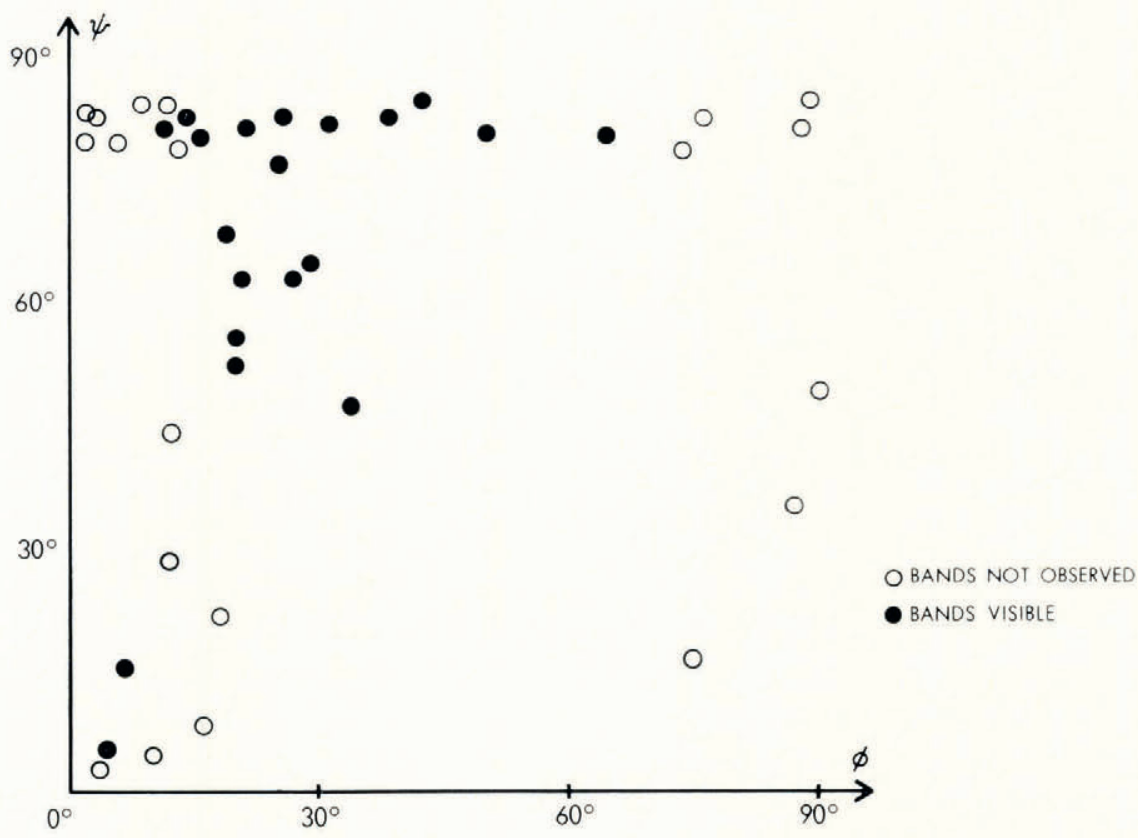

Fig. 6. Visibility of interference bands as a function of the inclination of the optic axis $\phi$ and the angle between the optic axis and the axis of compression $\psi$.

\section{(a) The stress-optical hypothesis}

If, as seems probable, the stresses were not uniformly distributed throughout the deformed ice crystals, it is clear that there could well be variations in the extinction position across a specimen. In particular, non-uniform stresses within a single lamella could lead to differences in the extinction position across it. Alternatively, there could be variations in the extinction position between neighbouring lamellae as a result of differences in the stresses applied to them. In either case a series of light and dark bands parallel to the slip traces would be seen between crossed polarizers. However, if the deformation was not too great, the variations in the extinction position might be expected to be small and the interference bands to only be clearly visible near the original extinction position, i.e. when the specimen appears almost completely dark. 
The spacing of such interference bands would be determined by the fluctuations in the non-uniform stress and would therefore be essentially independent of the wavelength of the light used to view them, in agreement with our observations. In the first case, however, the spacing would be less than the spacing of the slip lines contrary to our observations. This hypothesis therefore requires variations in the extinction position between neighbouring lamellae.

The hypothesis does not, however, readily account for the change in direction of the interference bands with variations in the thickness of the specimen, since this would require the extinction position to be dependent on the thickness of the specimen.

The strongest evidence against the validity of this hypothesis - at least, in the form in which it is being considered at present-is that the interference bands were not normally seen when the lamellae were viewed edge on, whereas one would have expected variations in the extinction position between neighbouring lamellae to be most clearly seen in this orientation (cf. Nye's (1949, r950) observations on silver chloride).

\section{(b) The misorientation hypothesis}

Consider a small part of the deformed crystal as represented in Figure 7 (the overlapping regions are so small that they can be neglected). In the misorientation hypothesis it is postulated that there are slight changes in the orientation of the crystal lattice on passing from one slip lamella to another.

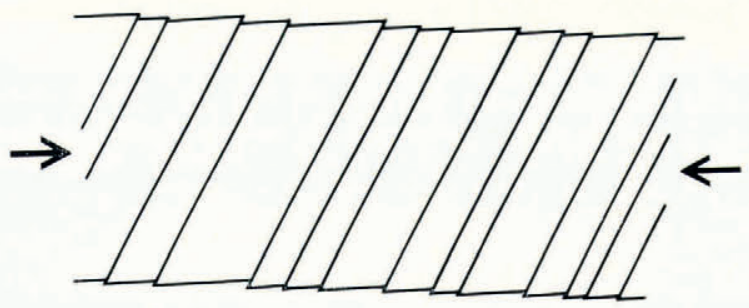

Fig. 7. Diagram of an ice crystal deformed by compression.

If the total deformation is not too large, the angles between the principal axes of each of the lamellae and the principal axes of the undeformed crystal are small and since no net rotation of the extinction position was normally observed, it is reasonable to assume that the misorientations are evenly distributed about the original extinction position. Further, since the misorientations are small, light is propagated through a deformed specimen with negligible deviation and therefore a plane polarized beam of light entering the crystal will pass through what are, in effect, a series of linear retarders. On emerging it will, in general, be elliptically polarized, but because the lamellae are inclined to the axis of viewing, rays of light passing through adjacent parts of the specimen will pass through slightly different combinations of linear retarders and emerge with slightly differing polarizations. This will in turn produce slight variations in the intensity transmitted through the analyser.

It is clear that, as in the case of the stress-optical hypothesis outlined above, the variations in intensity will be small and therefore only clearly visible near the original extinction position. It is also clear that since the phenomenon depends on the orientation of the slip lamellae, the spacing of the light and dark interference bands will be essentially determined by them and be independent of the wavelength of light.

In contrast to the stress-optical hypothesis, however, the spacing of the interference bands can be expected to be rather greater than the spacing of the slip lines, since the fluctuations in intensity are a result of the fact that light traverses different slip lamellae. 
This hypothesis can also account for changes in the direction of the interference bands in specimens of variable thickness, because the precise state of polarization of the emergent light depends on the number and thickness of the slip lamellae which it traverses.

Finally, this hypothesis accounts qualitatively for the fact that the interference bands are not normally visible when the optic axis is nearly parallel to or nearly perpendicular to the direction of viewing. In the first case, the interference bands are not seen because the lamellae are very nearly optically isotropic and very large differences in their orientation are necessary to produce a visible effect. In the second case, the lamellae are being viewed almost end on and therefore in many cases the light will only traverse one or two different lamellae, whereas it is an essential feature of the misorientation hypothesis that the light traverses a number of lamellae.

In view of this qualitative agreement between the observations and the misorientation hypothesis, it will now be examined in more detail.

(c) Mathematical analysis of the misorientation hypothesis

Using the calculus introduced by Jones (194I[a], [b]; Hurwitz and Jones, I94I), the set of $n$ lamellae in series through which any given ray passes can be represented by a single $2 \times 2$ matrix $M$ which is the product of the matrices representing each of the individual lamellae. In this case each lamella can be represented by a matrix $R_{j}^{\prime}$ which is the product of three matrices.

$$
R_{j}{ }^{\prime}=S\left(\rho_{j}\right) R_{j} S\left(-\rho_{j}\right)
$$

where $R_{j}$ is the matrix of the element referred to its own principal axes and $\rho_{j}$ is the angle between these axes and the principal axes of the undeformed crystal. The forms of the $S$ and $R$ matrices have been defined above (Equations (2) and (4) in section 2.3).

By assuming $\rho_{j}$ is small and neglecting the second order of small quantities it can be shown that

$$
R_{j}^{\prime}=\left[\begin{array}{cc}
\exp \left(\mathrm{i} \gamma_{j}\right) & 2 \mathrm{i} \rho_{j} \sin \gamma_{j} \\
2 \mathrm{i} \rho_{j} \sin \gamma_{j} & \exp \left(-\mathrm{i} \gamma_{j}\right)
\end{array}\right]
$$

where $2 \gamma_{j}$ is the retardance of the $j$ th element in the series. The sine terms must be retained because it is not valid to assume that the retardances of the individual elements are small. A sheet of ice with a thickness which is comparable with the thickness of the lamellae deduced from the observed spacing between the slip bands $(c .100 \mu \mathrm{m})$ would have a retardance of about $\pi / 2$.

The terms of the matrix $M$ representing all the lamellae traversed by a given ray can be evaluated by taking the product of a series of matrices of the form given by Equation (12).

$$
M=R_{n}{ }^{\prime} R_{n-1}{ }^{\prime} \ldots R_{2}^{\prime} R_{\mathrm{I}}{ }^{\prime}=\left[\begin{array}{ll}
m_{11} & m_{12} \\
m_{21} & m_{22}
\end{array}\right] .
$$

Neglecting terms of the order $\rho_{j}^{2}$, the matrix elements are found to be as follows:

$$
\begin{aligned}
& m_{11}=\exp (+\mathrm{i} \Gamma), \\
& m_{22}=\exp (-\mathrm{i} \Gamma), \\
& m_{12}=2 \mathrm{i} \sum_{j=1}^{n} \rho_{j} \sin \gamma_{j} \exp \left(\mathrm{i} \Gamma_{j}\right), \\
& m_{21}=2 \mathrm{i} \sum_{j=1}^{n} \rho_{j} \sin \gamma_{j} \exp \left(-\mathrm{i} \Gamma_{j}\right)
\end{aligned}
$$

where $2 \Gamma$ is the retardance of the whole crystal 
and

$$
\begin{aligned}
& \Gamma_{j}=\sum_{k=j+1}^{n} \gamma_{k}-\sum_{k=1}^{j-1} \gamma_{k} \quad \text { for } j=2 \text { to } n-\mathrm{I}, \\
& \Gamma_{1}=\sum_{2}^{n} \gamma_{k}, \\
& \Gamma_{n}=\sum_{1}^{n-1} \gamma_{k} .
\end{aligned}
$$

Now consider the case in which the analyser and polarizer are set so as to give extinction for the undeformed crystal, i.e. their axes are parallel to the reference axes. Then the vector $E_{\mathrm{t}}$ representing the elliptically polarized beam emerging from the crystal is given by

$$
E_{\mathrm{t}}=\left[\begin{array}{l}
E_{x} \\
E_{y}
\end{array}\right]=\left[\begin{array}{ll}
m_{11} & m_{12} \\
m_{21} & m_{22}
\end{array}\right]\left[\begin{array}{l}
\mathrm{I} \\
\mathrm{o}
\end{array}\right]=\left[\begin{array}{l}
m_{11} \\
m_{21}
\end{array}\right] .
$$

If the crystal were undeformed $m_{21}$ would be exactly zero, $m_{11}$ would be equal to $\exp (\mathrm{i} \Gamma$ ) and the emergent beam would be plane polarized.

In the case of the deformed crystal, the expressions derived above show that

$$
\begin{aligned}
& E_{x}=\exp (\mathrm{i} \Gamma), \\
& E_{y}=2 \mathrm{i} \sum_{j=1}^{n} \rho_{j} \sin \gamma_{j} \exp \left(-\mathrm{i} \Gamma_{j}\right) .
\end{aligned}
$$

Because the misorientations $\rho_{j}$ are small and evenly distributed about the mean extinction position, the component transmitted by the analyser $E_{y}$ is small. Hence it is clear that the emergent beam is very nearly plane polarized parallel to the $x$-axis, but with a component $E_{y}$ which is, in general, not equal to zero. As one moves across the crystal this component will fluctuate and hence the ellipticity of the emergent beam will also fluctuate.

The general behaviour of the component $E_{y}$ is not easily visualized and therefore the state of polarization of the emergent beam has been evaluated in detail for a special case, illustrated by Figure 8 . In this model of a deformed crystal it is postulated that the principal axes of

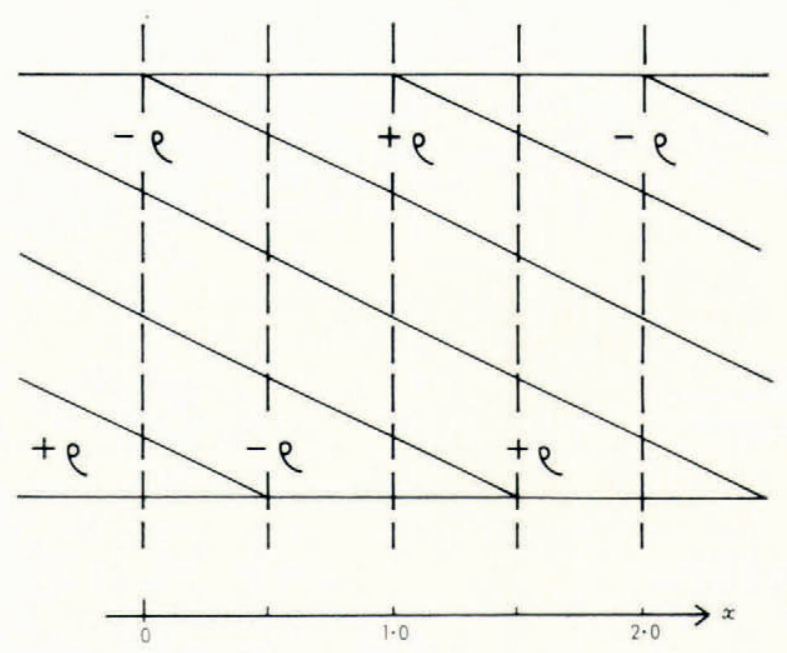

Fig. 8. Idealized model of a deformed ice crystal. 
alternate lamellae are inclined at angles of $+\rho$ and $-\rho$ to the reference axes (where $\rho$ is small) and that the lamellae are all of equal thickness.

As shown above, when the axes of the polarizer and analyser are set parallel to the reference axes, the only component of the matrix representing the crystal which affects the intensity of light transmitted through the analyser is $m_{2 \mathrm{I}}$. The sum representing this term in Equation (14) can be rewritten in three parts representing the contributions of the first and last lamellae traversed and the intervening lamellae,

i.e.

$$
m_{2 \mathrm{I}}=2 \mathrm{i}\left[A_{\mathrm{I}}+A_{n}+A\right]
$$

where

$$
\begin{aligned}
A_{\mathrm{I}} & =\rho_{\mathrm{I}} \sin \gamma_{\mathrm{I}} \exp \left(-\mathrm{i} \Gamma_{\mathrm{I}}\right), \\
A_{n} & =\rho_{n} \sin \gamma_{n} \exp \left(-\mathrm{i} \Gamma_{n}\right), \\
A & =\sum_{j=2}^{n-1} \rho_{j} \sin \gamma_{j} \exp \left(-\mathrm{i} \Gamma_{j}\right),
\end{aligned}
$$

with

$$
\gamma_{j}=\gamma \text { if } j \neq \mathrm{I} \text { or } n
$$

and

$$
\rho_{j}=(-\mathrm{I})^{j} \rho \text {. }
$$

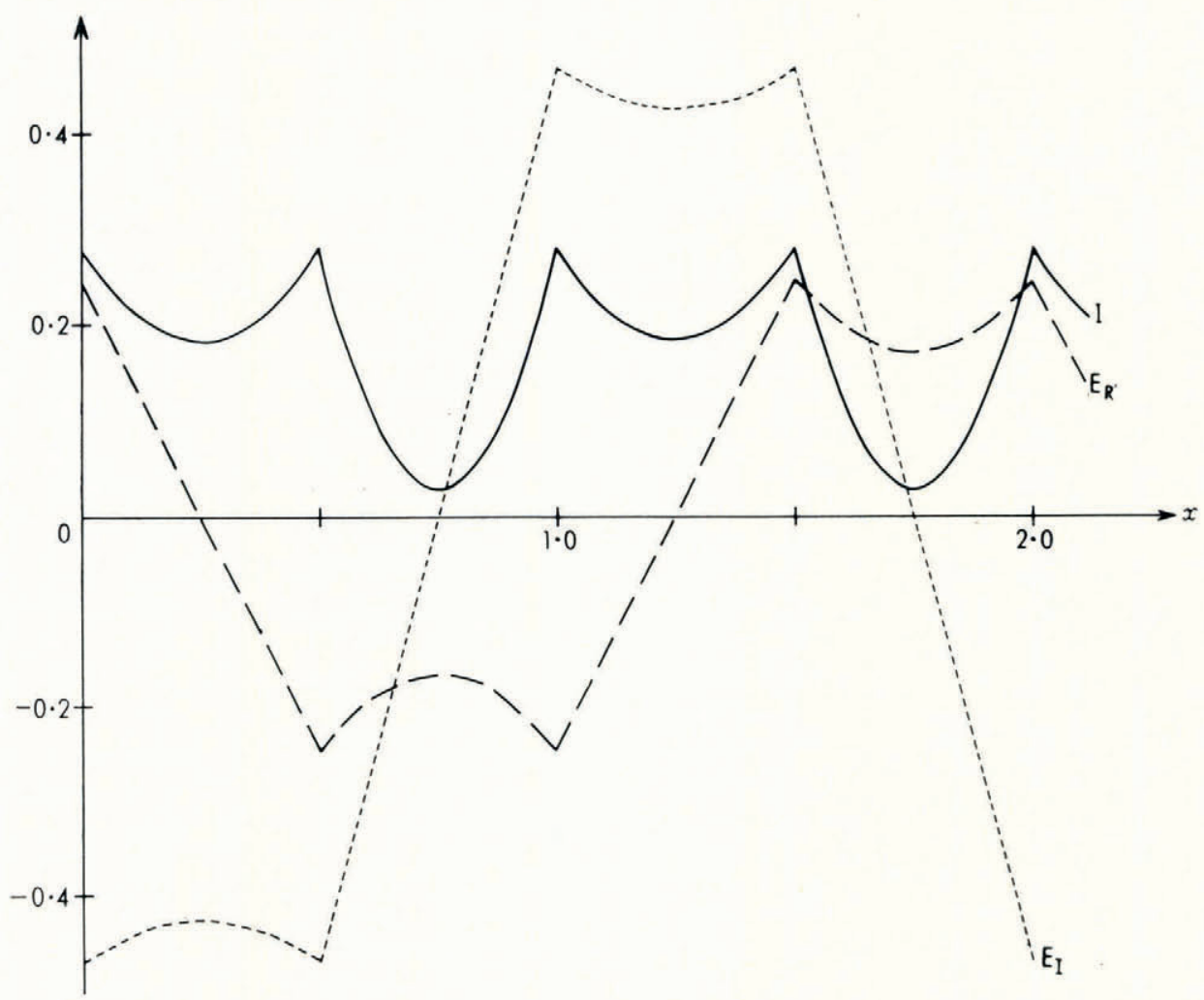

Fig. 9a. The amplitudes of the real and imaginary components, $E_{\mathrm{r}}$ and $E_{\mathrm{i}}$, and the resultant intensity $I$ of light transmitted through an idealized model of a deformed ice crystal.

Case 1 : inclination of optic axis $=25^{\circ}$, inclination of polarizers $=o^{\circ}$.

The horizontal axis is the distance across the crystal $x$, measured in relation to the slip-band spacing (see Fig. 8). The vertical axis gives the amplitudes in units of $\rho E_{0}$ and the intensity in units of $\rho^{2} I_{0}$, where $E_{0}$ and $I_{0}$ are the amplitude and intensity of the incident light. 
With these conditions

where

$$
\begin{aligned}
A_{\mathrm{I}} & =-\rho \sin \gamma_{\mathrm{I}} \exp \left\{-\mathrm{i}\left(\gamma_{n}+m \gamma\right)\right\}, \\
A_{n} & =(-\mathrm{I})^{n} \rho \sin \gamma_{n} \exp \left\{-\mathrm{i}\left(\gamma_{\mathrm{I}}+m\right)\right\}, \\
A & =-\rho \tan \gamma f \exp \left\{-\mathrm{i}\left(\gamma_{n}-\gamma_{\mathrm{I}}\right)\right\}, \\
f & =\left\{(-\mathrm{I})^{m} \exp (\mathrm{i} m \gamma)-\exp (-\mathrm{i} m \gamma)\right\} / 2 \\
m & =n-2 .
\end{aligned}
$$

and

These expressions have been evaluated numerically for several different cases.

Case I

$$
\begin{array}{ll}
\text { Thickness of crystal } & =2.5 \mathrm{~mm} \\
\text { Thickness of lamellae } & =0.1 \mathrm{~mm} \\
\text { Inclination of optic axis } & =25^{\circ} \\
\text { Inclination of polarizers to reference axes } & =0^{\circ}
\end{array}
$$

With these conditions each ray traverses a total of ro.5 lamellae and the spacing of the slip lines is o. I I mm. The intensity is then as shown in Figure ga.

It is clear from the diagram that the intensity fluctuates with a wavelength equal to the spacing between the slip lines, not several times that spacing, as found by observation and suggested by the qualitative analysis given above. The diagram also shows, however, that the real and imaginary components have a periodicity double the spacing between the slip lines and we therefore suggest that the shorter periodicity is only a result of the highly symmetrical case under consideration.

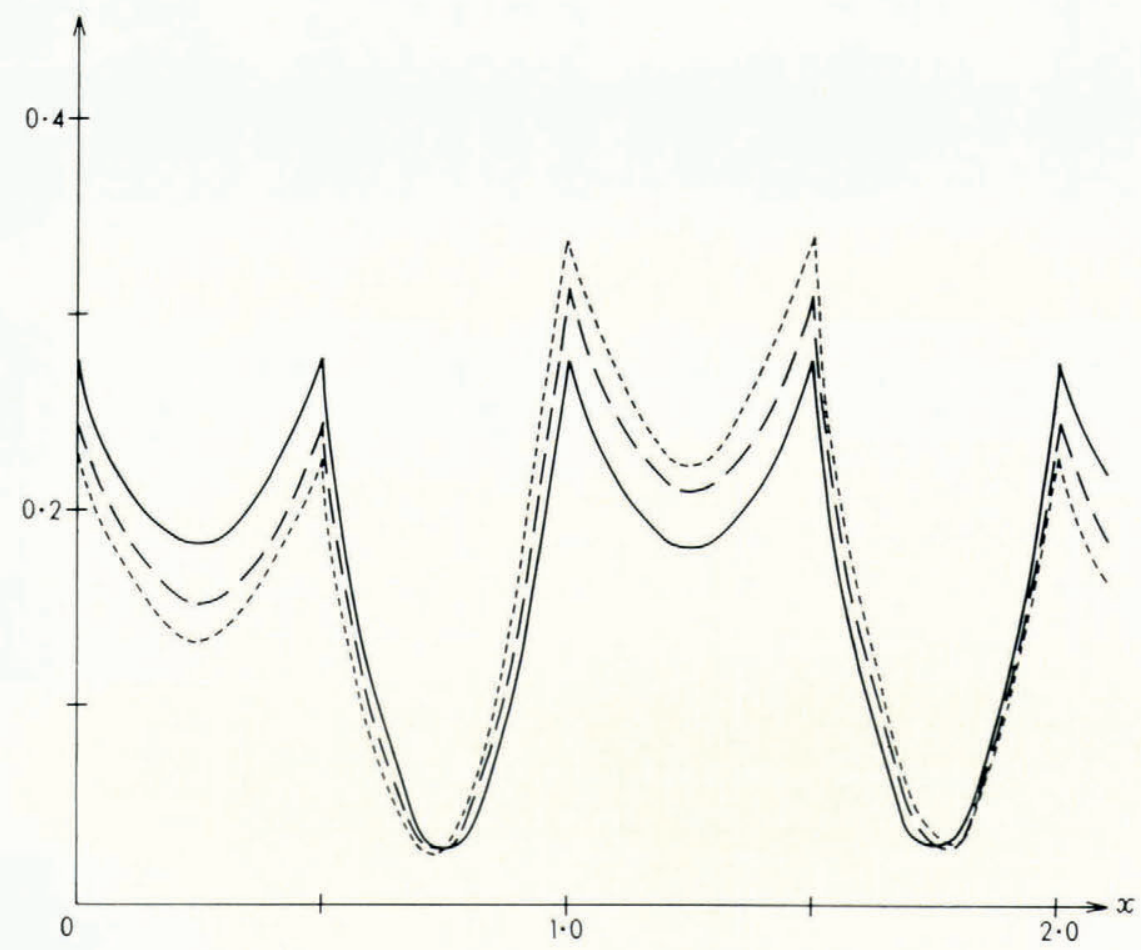

Fig. 9b. Intensities plotted as in Figure ga.

Case 2: inclination of optic axis $=25^{\circ}$, Inclination of polarizers:-

$$
-0^{\circ},-1.72^{\circ} \text { and } \cdots \cdots \cdots 2.36^{\circ} \text {. }
$$


Case 2

Thickness of crystal and lamellae and inclination of optic axis as in Case $\mathrm{r}$.

Variable inclination of polarizers from $0^{\circ}$ to $3^{\circ}$.

With these conditions the intensities are as shown in Figure $9 \mathrm{~b}$. This diagram shows that rotating the polarizers decreases the symmetry of the intensity distribution across the specimen, giving it a periodicity double the slip band spacing, and that this effect becomes more marked as the angle between the axes of the polarizers and the reference axes increases.

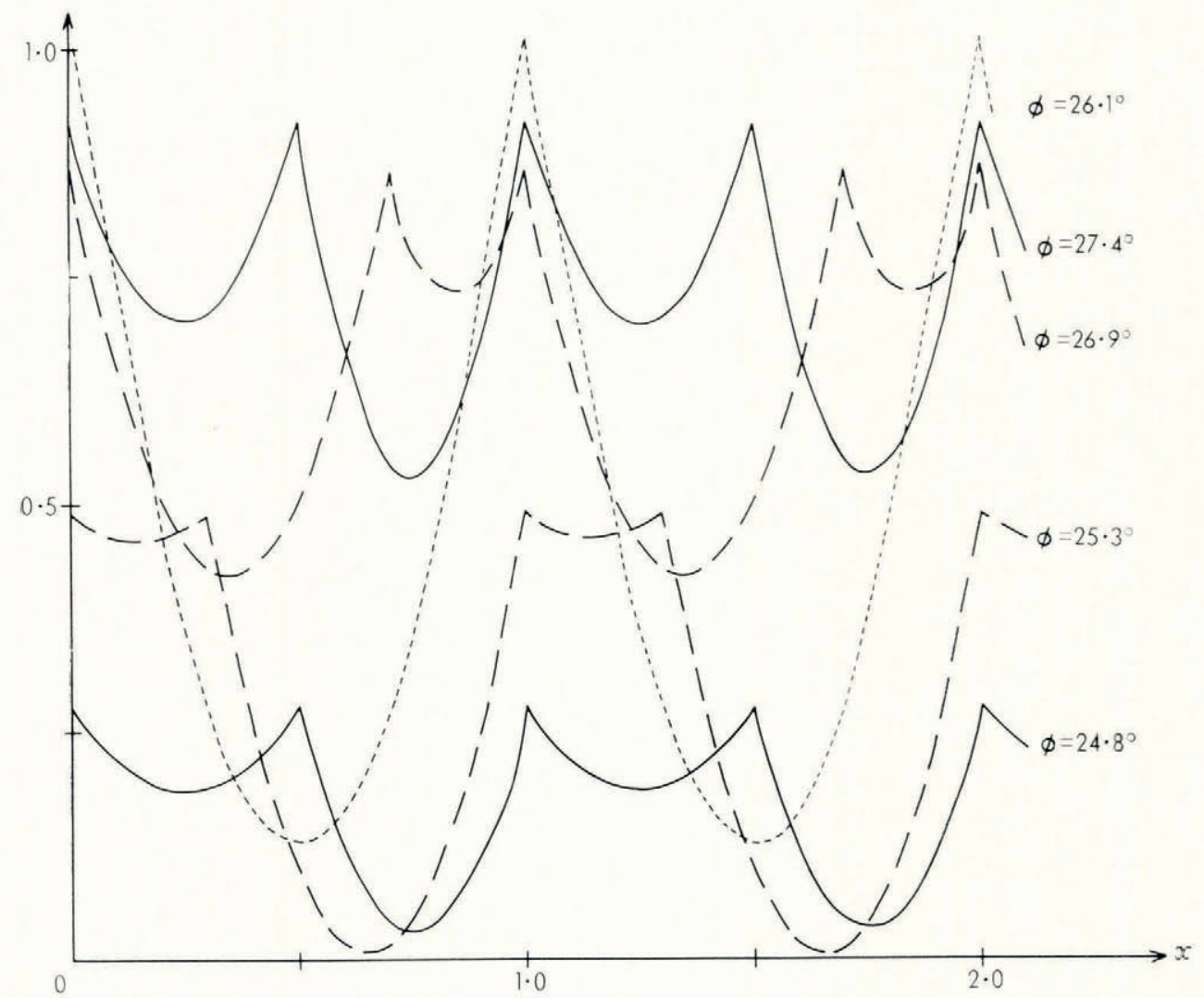

Fig. 9c. Intensities plotted as in Figure ga.

Case 3: inclination of polarizers $=0^{\circ}$, inclination of optic axis $=24.8^{\circ}$ to $27.4^{\circ}$ as shown.

Case 3

Thickness of crystal and lamellae as in cases $\mathrm{I}$ and 2.

Inclination of polarizers to references axes $=0^{\circ}$.

Variable inclination of optic axis from 24.8 to $26 . \mathrm{I}^{\circ}$.

With these conditions the number of lamellae traversed varies from ro.5 to I I.5. The resultant intensity distribution is shown in Figure 9c.

This diagram also shows that the transmitted intensity has a wavelength twice the slipband spacing, except when the number of lamellae traversed is exactly $n+0.5$, where $n$ is an integer. 
The model was also used to compute the maximum intensity transmitted as a function of the inclination of the optic axis. The result is plotted in Figure 10 and clearly shows that the interference bands will not be seen when $\phi \geqslant 70^{\circ}$. On the other hand, this graph indicated that interference bands may be seen when $\phi \leqslant \mathrm{I} 5^{\circ}$, contrary to our observations. It will be seen, however, that when $\phi$ is as small as this, only a few bands are traversed by each ray, even in the highly symmetrical model under consideration. In a real crystal the slip lamellae would not be expected to have either uniform thicknesses or equal misorientations and therefore it is not too surprising to find that in order for interference bands to be visible, the average number of lamellae traversed by each ray must be rather greater than the model would suggest.

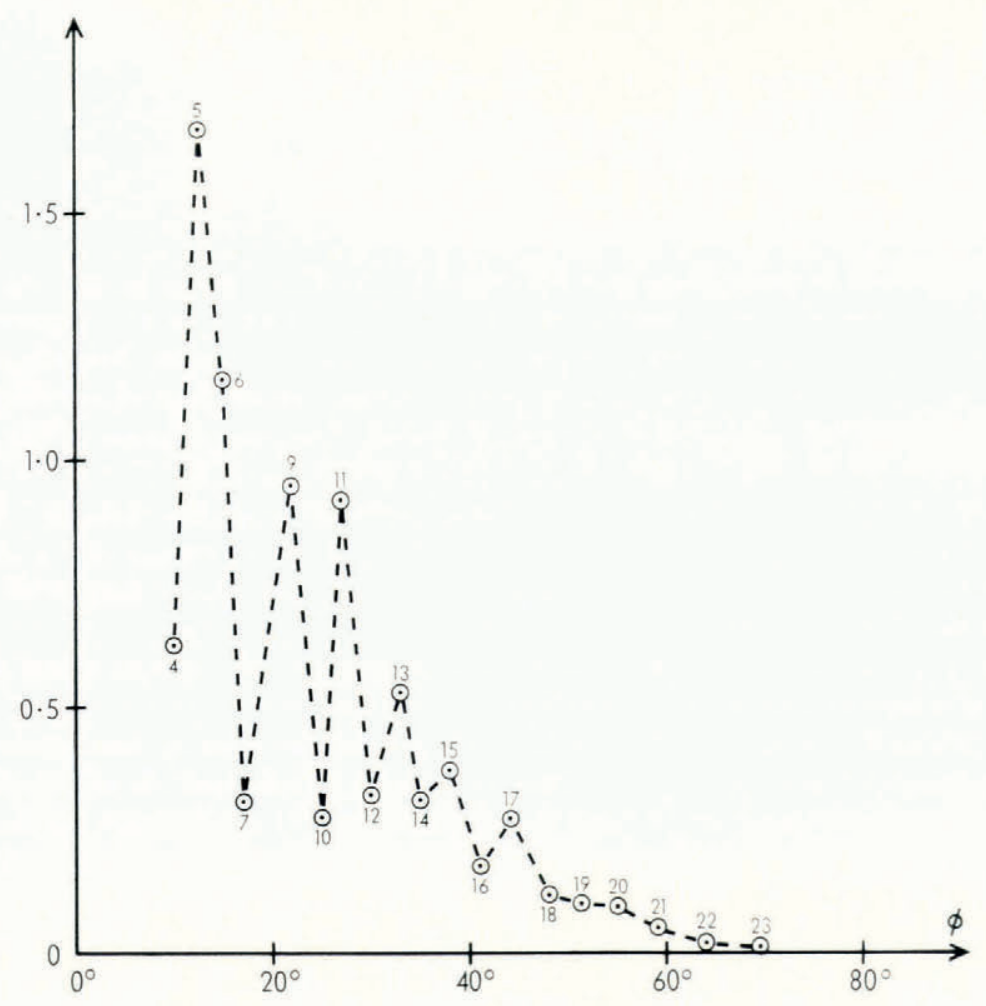

Fig. 10. Maximum intensity transmitted (in units of $\rho^{2} I_{0}$ ) as a function of the inclination of the optic axis $\phi$. The small numbers on the graph give the number of lamellae traversed $(=n+0.5)$.

\section{Discussion}

The evidence presented above favours the misorientation hypothesis. That is to say, it supports the suggestion that the individual lamellae produced by deformation are in slightly different crystallographic orientations. Because ice is a uniaxial crystal, however, no optical change could be observed if the lamellae were merely rotated with respect to each other about the $c$-axis and it is therefore essential to the theory that the direction of the $c$-axis should change from lamella to lamella.

A misorientation of this kind cannot be produced by the movement of dislocations whose Burgers vectors lie in the basal plane (which account for the majority of the slip in ice), but requires that dislocations with Burgers vectors having components parallel to the $c$-axis 
should accumulate on, or near, the active glide planes. The existence of such dislocations in ice has been inferred by a number of workers (e.g. Gold, I963; Muguruma and Higashi, 1963; Levi and others, I965) and we suggest that the present work also indicates that they do exist and make an active contribution to the deformation.

The degree of misorientation can be estimated from the results derived above. Figures 9 and Io show that the amplitude of the intensity variations predicted by our model is of the order of $0.5 \rho^{2} I_{0}$. This can be compared with intensity which is transmitted when the analyser and polarizer are rotated through an angle $\theta$ away from the extinction position for an undeformed crystal. In this case

$$
E_{\mathrm{r}}=A S(\theta) M S(-\theta) E_{\mathrm{i}}
$$

where $M$ is the matrix for an undeformed crystal and is given by

$$
M=\left[\begin{array}{cc}
\mathrm{e}^{\mathrm{i} \Gamma} & \mathrm{o} \\
\mathrm{o} & \mathrm{e}^{-\mathrm{i} \Gamma}
\end{array}\right] .
$$

From Equations (I8) and (I9), it follows that

$$
I=I_{0} \sin ^{2} 2 \theta \sin ^{2} \Gamma \text {. }
$$

Experiment shows that the variations in intensity in the interference bands are comparable with the variation produced by rotating the polarizers through $\frac{1}{4}$ to $I^{\circ}$. Assuming $\theta$ to be small, Equation (20) shows that $I$ is of the order $0.5 \theta^{2} I_{0}$. Hence $\rho \approx \theta$. Strictly $\rho$ is the angle between the projection of the $c$-axis on to a plane normal to the axis of viewing, but apart from the occasional special case, it will not differ greatly from the change in the direction of the $c$-axis.

We conclude, therefore, that there are tilt boundaries between the lamellae in which the angle of tilt is of the order of $\frac{1}{4}^{\circ}$ to $\mathrm{I}^{\circ}$. This is of the same order of magnitude as the low angle tilt boundaries observed in other materials and as the sub-divisions of larger tilt boundaries observed in ice by Nakaya (1958).

Finally, let us consider how the stress system acting on the crystal could produce tilt boundaries of the type required by the theory.

The most probable Burgers vector with a component parallel to the $c$-axis is $c\langle 000 \mathrm{I}\rangle$, since this is the shortest lattice vector not lying in the basal plane and the elastic strain energy of a dislocation is proportional to the square of the length of its Burgers vector. Such dislocations will probably glide on either $\left\{\mathrm{I}_{\overline{\mathrm{I}} \mathrm{O} O}\right\}$ or $\left\{\mathrm{II}_{\overline{2}} \mathrm{O}\right\}$ planes, although they could glide on any plane normal to the basal plane. A Burgers vector parallel to the $\left\langle\mathbf{1} \overline{1}_{2} 3\right\rangle$ direction is also possible, but seems less likely.

Now when a crystal is compressed along an axis which is inclined to the $c$-axis, there will be a resolved shear stress both on the basal plane and also on these planes. This means that dislocations of this type will experience a force tending to make them glide in a direction normal to the basal plane. It is clear, however, that the majority of deformation in ice takes place on localized basal planes which must presumably contain a large number of dislocations with Burgers vectors of the type $\frac{1}{3} a\langle\operatorname{I} \mathbf{2} \overline{2} 0\rangle$. We therefore visualize that dislocations with their Burgers vectors parallel to the $c$-axis will be generated between the active basal glide planes and glide until they pile-up against the dislocations on the active planes.

We suggest that they are generated, because it seems unlikely that there would be a sufficient number of "grown-in" dislocations to account for the observed misorientation and also because the interference bands become more marked as the deformation continues, presumably as a result of increasing misorientation between the lamellae. The resultant tilt will depend on the sum of the Burgers vectors of all the dislocations piled up against a glide band can be expected to vary in a random manner from glide band to glide band. The postulated array of dislocations is indicated schematically in Figure $\mathrm{I}$. 


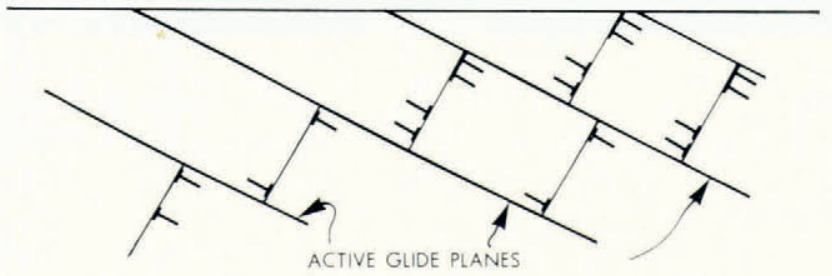

Fig. II. A possible network of dislocations in a compressed ice crystal.

\section{Summary and conclusions}

In the experiments described in this paper, single crystals of ice have been viewed between crossed polarizers in order to study changes which occur when they are deformed by compression.

Some interference bands were observed at grain boundaries produced by kinking. These bands can be accounted for by the changes in the orientation of the optic axis across the boundary and the spacings between them can be predicted with the aid of Jones' calculus, which is a powerful tool for the analysis of such systems. Irregularities in their spacings are a measure of irregularities in the grain boundary.

Jones' calculus has also been applied to a study of interference bands which were observed parallel to the trace of the basal plane on the surface of many of the deformed specimens. It appears probable that these bands are a result of slight misorientations between adjacent slip lamellae. If this interpretation is correct, then it implies that there is some deformation produced by the movement of dislocations with non-basal Burgers vectors, probably parallel to the $c$-axis, $\langle\mathrm{OOOI}\rangle$, or perhaps the $\left\langle\mathrm{II}_{2} 3\right\rangle$ direction. Although it is clear that any mechanism involving such dislocations only makes a small contribution to the total deformation, it may nevertheless be significant for the understanding of creep phenomena and some of the textures of deformed polycrystalline ice observed in nature.

\section{Acknowledgements}

The experimental part of the work described in this paper was carried out whilst the authors were at the Imperial College of Science and Technology, London. We wish to acknowledge the assistance given by staff of the college, especially by Dr A. Thetford who first drew our attention to the use of Jones' calculus for optical systems. At this time one of us (C. J. R.) was supported by a Research Studentship from the Department of Scientific and Industrial Research, and this is also gratefully acknowledged.

We should also like to thank Dr B. J. Mason, both for his personal interest in this work and, as Director-General of the Meteorological Office, for his permission to publish this paper.

MS. received 8 July 1970

\section{REFERENCES}

Bartlett, J. T., and Readings, C. J. 1968. Some optical effects in deformed single crystals of ice. Union de Géodésié et Géophysique Internationale. Association Internationale d'Hydrologie Scientifique. Assemblée générale de Berne, 25 sept.7 oct. 1967. [Commission de Neiges et Glaces.] Rapports et discussions, p. 316-25.

Gold, L. W. 1963. Deformation mechanisms in ice. (In Kingery, W. D., ed. Ice and snow; properties, processes, and applications: proceedings of a conference held at the Massachusetts Institute of Technology, February I2-I6, I962. Cambridge, Mass., The M.I.T. Press, p. 8-27.)

Higashi, A., and Sakai, N. 196r. Movement of small angle boundary of ice crystal. Fournal of the Physical Society of Japan, Vol. i6, No. i 1, p. 2359-6o. 
Hurwitz, H., jr., and Jones, R. C. 1941. A new calculus for the treatment of optical systems. II. Proof of three general equivalence theorems. Fournal of the Optical Society of America, Vol. 31, No. 7, p. 493-99.

Jones, R. C. 194I[a]. A new calculus for the treatment of optical systems. I. Description and discussion of the calculus. Fournal of the Optical Society of America, Vol. 31, No. 7, p. 488-93.

Jones, R. C. 194I[b]. A new calculus for the treatment of optical systems. III. The Sohncke theory of optical activity. Fournal of the Optical Society of America, Vol. 31, No. 7, p. 500-03.

Levi, L., and others. 1965 . Experimental study of non-basal dislocations in ice crystals, by L. Levi, E. M. de Achaval and E. Suraski. Journal of Glaciology, Vol. 5, No. 41, p. 691-99.

Muguruma, J., and Higashi, A. 1963 . Observation of etch channels on the (ooor) plane of ice crystals produced by non-basal glide. Fournal of the Physical Society of Japan, Vol. 18, No. 9, p. 1261-69.

Nakaya, U. 1958. The deformation of single crystals of ice. Union Géodésique et Géophysique Internationale. Association Internationale d'Hydrologie Scientifique. Symposium de Chamonix, I6-24 sept. 1958, p. 229-40.

Nye, J. F. 1949. Plastic deformation of silver chloride. I. Internal stresses and the glide mechanism. Proceedings of the Royal Society, Ser. A, Vol. 198, No. 1053, p. 190-204.

Nye, J. F. 1950. Plastic deformation of silver chloride. II. Photoelastic study of the internal stresses in glide packets. Proceedings of the Royal Society, Ser. A, Vol. 200, No. 106o, p. 47-66.

Readings, C. J., and Bartlett, J. T. 1968. Slip in single crystals of ice. Journal of Glaciology, Vol. 7, No. $5^{1}$, p. $479-9 \mathrm{I}$. 